\title{
Aortitis Associated to with Rheumatoid Arthritis: A Challenginge Rheumatoid Vasculitis Presentation: A Case Report
}

\author{
Zeineb Teyeb $^{1 *}$, Mohamed Ben Salah ${ }^{2}$, Lobna Kharrat ${ }^{1}$, Imen Abdellali ${ }^{1}$, Taieb Jomni ${ }^{1}$ and Mohamed Hedi Douggui ${ }^{1}$ \\ ${ }^{1}$ Department of Internal Medicine, Internal Security Forces Hospital, Tunis, Tunisia \\ ${ }^{2}$ Department of Orthopedic Surgery, Charles Nicolle Hospital, Tunis, Tunisia
}

${ }^{\star}$ Corresponding author: Zeineb Teyeb, Department of Internal Medicine, Internal Security Forces Hospital, Tunis, Tunisia

Received: February 03, 2021; Accepted: February 08, 2021; Published: February 15, 2021

\begin{abstract}
Rheumatoid Vasculitis (RV) is a rare but serious extra-articular manifestation of Rheumatoid Arthritis (RA). Its varied clinical presentation makes it hard to diagnose and treat. Hereby we describe a case of an aortitis revealing RV which is a rare presentation of a rare complication of RV. A 56-year-old man with rheumatoid arthritis treated with methotrexate presented with fever, chest pain and arthritis. Blood tests revealed inflammatory syndrome associated with cholestasis. The diagnosis of pericarditis associated with aortitis was retained. Cholestasis was mostly due to methotrexate. The patient was treated with cyclophosphamide pulses and high doses of prednisolone. The patient was in complete remission of articular and extra-articular manifestations after two months of treatment.
\end{abstract}

Keywords: Rheumatoid arthritis, Rheumatoid vasculitis, Aortitis, Immunosuppressive therapy, Case report

\section{Introduction}

Rheumatoid Arthritis (RA) is a connective tissue disease predominantly affecting the joints. Extra-articular manifestations develop in up to $40 \%$ of cases [1], of which Rheumatoid Vasculitis (RV) is the most serious. The widespread vascular involvement, which effects not only the synovia but also other organs such as the skin, eye and nerves, can be life threatening. Mortality can reach up to $40 \%$ within five years of disease onset [2]. Fortunately, RV is a rare complication that occurs in $1-5 \%$ of RA patients [3]. It commonly affects small and medium blood vessels [4]. Large vessel vasculitis is unusual during RA. Hereby we describe a case of an aortitis revealing $\mathrm{RV}$ which is a rare presentation of a rare complication of $\mathrm{RV}$.

\section{Case Description}

\section{Patient Information and Clinical Findings}

A 54-year-old man, a North African policeman living in an urban environment with a personal history of smoking and pulmonary tuberculosis in 1991 was diagnosed in 2009 with seropositive and erosive RA associated with Sjögren's syndrome. He was being treated with methotrexate (25 mg/week). On November 2019, the patient presented to our hospital with fever, fatigue and chest pain that had started one week prior. Physical examination found a high temperature of $38.5^{\circ} \mathrm{C}$. Systolic blood pressure was $100 \mathrm{mmHg}$ and diastolic blood pressure was $60 \mathrm{mmHg}$ in both arms. He had tachycardia, with a heart rate of 115 beats per minute. There were no signs of heart failure and respiratory rate was normal. Mobilization of the wrists, elbows and shoulders was painful, with a swollen right wrist. He had a dislocation of the right ulnar styloid. He had rheumatoid nodules on the outer side of both elbows. There were no skin ulcerations no erythema nodosum. The rest of the physical examination was normal.

\section{Diagnostic Assessment}

An electrocardiogram showed atrial fibrillation with a heart rate of 115 beats per minutes associated with diffuse ST-segment elevation with upward concavity. Blood tests showed hyper leukocytosis at $11000 / \mathrm{mm}^{3}$, C-reactive Protein (CRP) levels of $117 \mathrm{mg} / \mathrm{l}$, an Erythrocyte Sedimentation Rate (ESR) of $118 \mathrm{~mm}$ and cholestasis with increased gamma glutamyl transferase and phosphatase alkaline levels of $199 \mathrm{UI} / \mathrm{L}$ (four times the normal rate) and $348 \mathrm{UI} / \mathrm{L}$ (five times the normal rate), respectively. Transaminase levels were normal (ALAT level of $35 \mathrm{UI} / \mathrm{L}$ and ASAT level of $25 \mathrm{UI} / \mathrm{L}$ ). A procalcitonin test was negative $(<0.5 \mu \mathrm{g} / \mathrm{L})$. Blood gas analysis was normal ( $\mathrm{pH}$ level of 7.40, $\mathrm{PaO} 2$ level of $90 \mathrm{mmHg}, \mathrm{PaCO} 2$ level of $42 \mathrm{mmHg}, \mathrm{HCO} 3-$ level of $25 \mathrm{mmol} / \mathrm{l})$.

Blood cultures were negative even for fungal agents. A chest X-ray showed a flask-shaped enlarged cardiac silhouette. Transthoracic echocardiography confirmed a non-compressive large posterior pericardial effusion. Abdominal ultrasound was normal. A thoracoabdominal Computed Tomography (CT) scan showed pericardial effusion with enhancement of the pericardium, compatible with pericarditis, and regular parietal hypodense circumferential concentric thickening of the aortic arch and supra aortic arterial trunk root, confirming aortitis (Figures 1 and 2). CT showed no bubbles in nor around the aorta wall. There was no mural thrombus nor aortic 


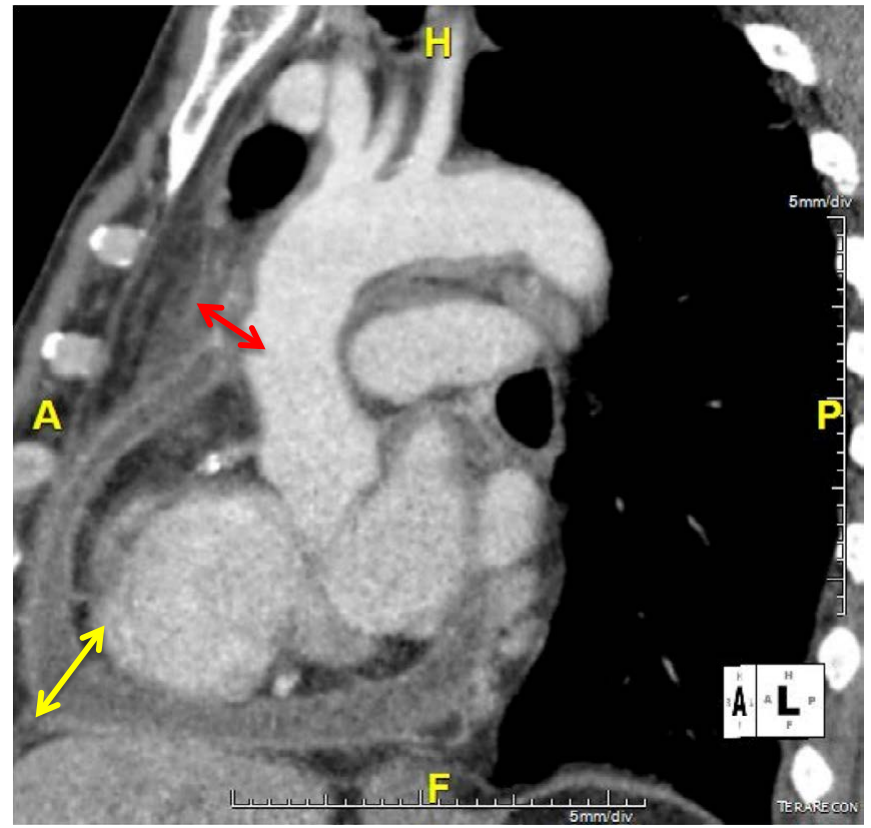

Figure 1: Thickening of the aorta (red arrow) associated with cardiac effusion (yellow arrow) in a sagittal chest CT.

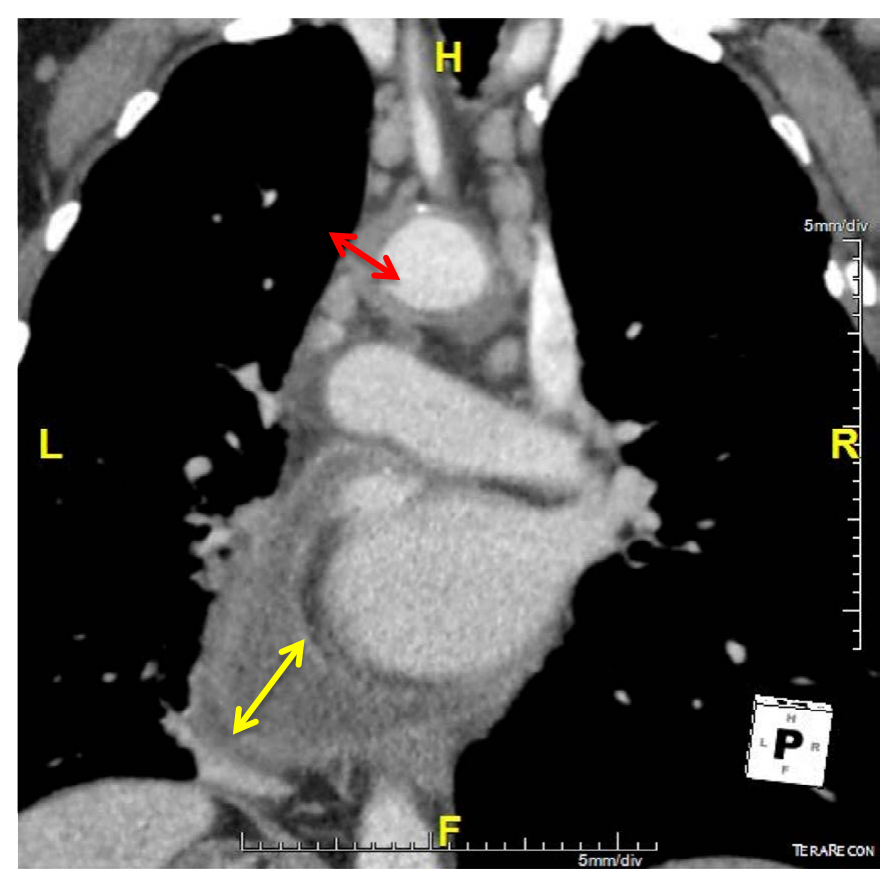

Figure 2: Thickening of the aorta (red arrow) associated with cardiac effusion (yellow arrow) in a frontal chest CT.

aneurysm nor signs of atherosclerosis. There was emphysema in pulmonary parenchyma but no evidence of active tuberculosis on chest-CT. Positron emission tomography was not perform due to its availability in our country.

A biopsy of the cardiac effusion was not possible due to its posterior location. The sputum test for Koch's bacillus was negative. Viral hepatitis B and C and syphilis serology were negative. Antinuclear antibodies were positive at 1/1200. Anti-LKM1, anti-CCP and rheumatoid factor levels were respectively of $1 / 80,40 \mathrm{IU} / \mathrm{ml}$ and 50
$\mathrm{IU} / \mathrm{ml}$. Immunoglobulin levels were normal (Immunoglobulin G level of $14.3 \mathrm{~g} / \mathrm{L}$, immunoglobulin $\mathrm{M}$ level of $2.08 \mathrm{~g} / \mathrm{L}$ and immunoglobulin A level of $1.8 \mathrm{~g} / \mathrm{L}$ ). Liver biopsy showed peliosis with no sign of autoimmune hepatitis nor auto-immune biliary cholangitis. The diagnosis of RV with large vessel and cardiac involvement was retained. Disease activity was evaluated as moderate by the DAS-28 CRP score.

\section{Therapeutic Intervention}

The patient was treated with a high dose of prednisone $(60 \mathrm{mg} /$ day) and IV pulses of $1000 \mathrm{mg}$ cyclophosphamide every month for six months. Treatment with methotrexate was stopped. Because of a personal history of pulmonary tuberculosis and the prescription of corticosteroids and cyclophosphamide in an epidemic country of tuberculosis, we administered prophylactic antitubercular therapy. The patient was also by treated with $200 \mathrm{mg} /$ day of amiodarone for the atrial fibrillation.

\section{Follow-Up and Outcomes}

The patient was apyretic after day 2 and their heartbeat became normal. Chest pain and articular manifestations decreased and disappeared after one month of treatment. CRP levels decreased to $12 \mathrm{mg} / \mathrm{L}$ after steroids and cyclophosphamide pulses. ESR became normal after two months of treatment. Cholestasis disappeared after one and a half months of methotrexate withdrawal.

Investigations of the cholestasis highlighted the iatrogenic involvement of methotrexate, confirmed by a report from the National Drug Safety Department in Charles Nicolle's Hospital of Tunis.

Cardiac echography showed the disappearance of the cardiac effusion at two months. A chest CT scan showed a significant regression of the vasculitis.

\section{Discussion}

The epidemiology of RV is hard to define. Heterogeneous clinical presentation, paucity of specific data to confirm the diagnosis and lack of a unanimous definition of RV are some of the reasons why it is hard to define. Many authors claim that RV can be observed in up to $5 \%$ of RA patients [5]. Our patient was 54 years old. The mean age of patients at diagnosis of aortitis in a literature review was $56 \pm 15.2$ years old [6].

RV commonly affects small and medium sized vessels. The skin is the most commonly damaged organ ( $90 \%$ of patients). The association of aortitis with RV is not widely recognized. However, many authors reported an association of RA with aortitis [6]. Our patient had a lesion typical of vasculitis of the aorta on his chest CT scan. Differential diagnoses were ruled out such as syphilis, tuberculosis or other infectious causes (negative sputum test for Koch's bacillus, negative serologies of viral hepatitis $\mathrm{B}$ and $\mathrm{C}$ and syphilis, negative blood cultures for bacteria or fongi).

Systemic erythematous lupus was unlikely. The patient didn't have any suggestive skin lesions nor pleurisy. The direct coombs test was negative. He didn't have any cytopenia and proteinuria was negative. Anti-DNAn, anti-Sm, anti-nucleosome, anti-histones were negative. Takayasu's arteritis was also unlikely. The patient had no upper or lower limbs claudication. He had no dizziness nor ocular symptoms. 
Blood pressure was normal and symmetrical. Peripheral pulses were present and symmetrical. The lesions of the aorta on CT scan was not suggestive of Takayasu's Arteritis.

Giant cell arteritis were excluded. The patient had no history of stroke, no headache nor ocular symptoms suggestive of Giant cell arteritis. Temporal artery was normal with a normal and symmetrical pulse. Aortitis was the only manifestation of RV in our patient. Other similar cases have been reported. In half of the cases, aortitis was isolated, with no other features of vasculitis [6]. In our patient, RV was revealed after 11 years of RA onset while the patient was treated with methotrexate and a low dose of corticosteroids. In a review of the literature, RV appeared after a mean disease duration of six years. Rheumatoid nodules were observed in up to half of patients with RV [6].

Our patient had poor articular manifestations with disease activity evaluated as moderate by the DAS- 28 score. The concept of 'burnt out' disease is described by many authors, consisting of the contrast between benign articular presentation and severe life-threatening RV [4]. This leads us to insist on actively screening this rare but fatal complication. RV typically occurs in long-standing seropositive and erosive RA, especially in males, smokers and patients with rheumatoid nodules or rheumatoid pericarditis [1,5]. Our patient had all these conditions.

There are no randomized controlled studies to guide the management of RV. However, treatment must be guided by the severity of organ involvement. High doses of corticosteroids and cyclophosphamide have been known to be the treatment of severe forms of RV such as aortitis [1,5-9]. Our patient had a good response to high doses of prednisolone and cyclophosphamide. Biotherapy such as TNF inhibitors, rituximab, abatacept and anakinra could be a good alternative $[4,10]$.

Another particularity of our patient is his liver injury. The patient had increased gamma glutamyl transferase and phosphatase alkaline levels with normal transaminases. This cholestasis is mostly due to methotrexate. This was confirmed by the complete normalization of liver enzyme levels after methotrexate withdrawal and the report from the drug safety department. However, our patient had Autoimmune Hepatitis (AIH) antibodies without any histological pattern of $\mathrm{AIH}$ and with normal levels of transaminase and immunoglobulins. There was not enough evidence to retain the diagnosis of AIH. Furthermore, surveillance of liver biology is recommended to assess the risk of developing AIH [11-13].

\section{Conclusion}

In the past years, the incidence of RV has decreased. Early diagnosis of RA, treat-to-target treatment strategies and the large use of methotrexate and biological molecules has improved the quality of life of RA patients [5]. Better management of the disease has led to a diminishing incidence of RV. However, clinical presentation remains unchanged. The mortality rate remains high, making RV a life-threatening condition that must be screened and treated early and aggressively. In addition, liver injury in RA patients varies from infectious (hepatitis B or C), toxic (paracetamol, methotrexate) and autoimmune.

\section{Data Availability}

All data underlying the results are available as part of the article and no additional source data are required.

\section{Consent}

Written informed consent for publication of their clinical details and images was obtained from the patient.

\section{Competing Interests}

No competing interests were disclosed.

\section{Grant Information}

The author(s) declared that no grants were involved in supporting this work.

\section{References}

1. Makol A, Crowson CS, Wetter DA, Sokumbi O, Matteson EL, et al. (2014) Vasculitis associated with rheumatoid arthritis: A case-control study. Rheumatology (Oxford, England) 53: 890-899. [crossref]

2. Turesson C, O'Fallon WM, Crowson CS, Gabriel SE, Matteson EL, et al. (2002) Occurrence of extrarticular disease manifestations is associated with excess mortality in a community based cohort of patients with rheumatoid arthritis. J Rheumatol 29: 62-67. [crossref]

3. Cojocaru M, Cohocaru IM, Chico B (2015) New insight into the rheumatoid vasculitis. Romanian J Intern Med Rev Roum Med Interne 53: 128-132. [crossref]

4. Kishore S, Maher L, Mjithia V (2017) Rheumatoid Vasculitis: A Diminishing Yet Devastating Menace. Current Rheumatology reports 19: 39. [crossref]

5. Makol A, Matteson EL, Warrington KJ (2015) Rheumatoid vasculitis: An update. Curr Opin Rheumatol 27: 63-70. [crossref]

6. Genta MS, Genta RM, Gabay C (2006) Systemic rheumatoid vasculitis: A review. Semin Arthritis Rheum 36: 88-98. [crossref]

7. Kaneko S, Yamashita H, Sugimori Y, Takahashi Y, Kaneko H, et al. (2014) Rheumatoid arthritis-associated aortitis: A case report and literature review. Springer Plus 3: 509. [crossref]

8. Scott DG, Bacon PA (1984) Intravenous cyclophosphamide plus methylprednisolone in treatment of systemic rheumatoid vasculitis. Am J Med 76: 377-384. [crossref]

9. Nanatsaki E, Mooney J, Scott DGI, Watts RA (2014) Systemic rheumatoid vasculitis in the era of modern Immunosuppressive therapy. Rheumatology (Oxford, England) 53: 145-152. [crossref]

10. Van der Bijl AE, Allaart CF, Van Vugt J, Van Duinen S, Breedveld FC (2005) Rheumatoid vasculitis treated with infliximab. J Rheumatol 32: 1607-1609. [crossref]

11. Gatselis NK, Zachou K, Koukoulis GK, Dalekos GN (2015) Autoimmune hepatitis, one disease with many faces: Etiopathogenetic, clinico-laboratory and histological characteristics. World J Gastroenterol 21: 60-83. [crossref]

12. Weinblatt ME, Tesser JR, Gilliam JH (1982) The liver in rheumatic diseases. Semin in Arthritis and Rheumatism 11: 399-405. [crossref]

13. Utiyama S, Zenatti K, Nóbrega H, Soares J, Skare T, et al. (2016) Rheumatic disease autoantibodies in autoimmune liver diseases. Immunol Invest 45: 566-573. [crossref]

Citation:

Teyeb Z, Salah MB, Kharrat L, Abdellali I, Jomni T (2021) Aortitis Associated to with Rheumatoid Arthritis: A Challenginge Rheumatoid Vasculitis Presentation: A Case Report. Integr J Orthop Traumatol Volume 4(1): 1-3. 